\title{
Una discusión sobre las condiciones de la reproducción campesina*
}

\author{
Vania Almeida Salles
}

In fact, as $I$ want to argue, the essential character of peasantry actually, derives from its being a class of survivors.

John Berger

Este teXto REÚNe UN CONJUNTo de tesis producidas colectivamente durante los últimos años, en el marco de la investigación sobre el campesinado en México. Aunque existen agudas discrepancias en cuanto a problemas clave de la interpretación de la cuestión campesina, que son puestas en evidencia a través del debate teórico político, creo que hay razonables niveles de consenso sobre a) la existencia de una integración subordinada del campesinado al sistema dominante y de condicionantes macrosociales que sufre la economía campesina como consecuencia de este modo de inserción; $b$ ) la existencia de iniciativas campesinas que, al generar diversos tipos de acciones tendientes a garantizar su supervivencia, influyen en el proceso de creación y manutención de espacios para la reproducción de los actores involucrados en estas acciones.

Basado en este marco general, el trabajo que presento a continuación plantea inicialmente los rasgos principales del contexto de la reproducción campesina, recalcando la necesidad de un enfoque que reconozca la existencia de diversos ámbitos de reproducción, que dan sentido a la definición del campesinado. En seguida analizo algunos condicionantes macrosociales que, al

* Quiero agradecer a Marielle Martínez y Orlandina de Oliveira que pacientemente discutieron la primera versión de este trabajo e hicieron valiosas sugerencias. 
regular el acceso a la tierra y a medios de producción, intervienen en los resultados del trabajo agrícola, el cual es estudiado como parte del conjunto de las actividades económicas que realizan las familias campesinas para sobrevivir. Señalo además que pesan sobre el trabajo agrícola factores naturales, generados por la especificidad de la producción en la agricultura, que influyen en la disposición del tiempo que se dedica a esta actividad. Finalmente indico que la formación del ingreso global se caracteriza por la "dispersión" productiva, y que en general las distintas labores realizadas por las familias campesinas exigen gran despliegue de trabajo, por la imposibilidad de lograr una combinación técnica adecuada entre trabajo y medios de producción. Se ilustra esta situación para la actividad agrícola con datos sobre volúmenes desiguales de productos que se obtienen en tierras de una misma extensión, pero con calidades diferentes y con distintos montos de gastos en insumos y tecnología.

Los campesinos y el contexto de su reproducción

La finalidad inmediata de la producción campesina es satisfacer las necesidades de consumo de los propios campesinos: los medios de vida y los elementos necesarios para producir constituyen los requerimientos de la reproducción del ciclo productivo. El consumo (en estos dos aspectos), en tanto que condición para la continua utilización del trabajo, es el elemento motivador de la producción.

La producción campesina, al usar principalmente la fuerza de trabajo familiar, parte de una cantidad de trabajo disponible (dada por el tamaño de la familiă, la edad y el sexo de sus miembros, el ciclo vital familiar, las formas de aprendizaje y las aptitudes reconocidas socialmente) que determina la posibilidad de ampliar o intensificar la producción agrícola, o de diversificar las labóres (Martínez y Rendón, 1983). La contratación de mano de obra ajena, a su vez, al intervenir en el monto de trabajo de que dispone, aparece como un elemento que contrarresta la rigidez original impuesta por las características de la familia (Torres, 1981).

A pesar de que en la economía campesina parte de los medios de producción y de los medios de vida (y en ciertos casos igual- 
mente la tierra) no provengan directamente del mercado (Bartra, Paz Lanz y Moguel, 1977), resulta difícil considerar el autoconsumo como categoría defmitoria del campesinado. Sea por el requerimiento de mercancía que consume y no produce, sea por la necesidad de vender fuerza de trabajo o productos, el campesino genera vínculos de distinta naturaleza con el mercado.

En tales situaciones, presentándose como vendedor de productos o de fuerza de trabajo, el campesino ofrece mercancías que al ser compradas se integran al mercado capitalista (de trabajo o de productos). En muy poco casos los campesinos se desplazan a las grandes ciudades para comerciar: el mercado local (representado por acaparadores, bodegas oficiales, etc.) constituye la instancia a la que acuden para vender, y por este mecanismo los productos de miles de campesinos se integran a la producción social. A partir igualmente del mercado, se abastecen de las mercancias que necesitan pero que no producen. Independientemente del costo individual de su producto, los campesinos reciben una remuneración que corresponde aproximadamente a los precios usuales, fijados socialmente. La naturaleza externa (y ajena) de los precios de mercado también se manifiesta en las compras hechas por los campesinos. A partir de la relación entre lo que recibieron por vender (inclusive la fuerza de trabajo familiar) y lo que gastarán para comprar, se establece su pauta de consumo posible, en cuanto a bienes que sólo pueden ser adquiridos en el mercado.

Por lo tanto se descarta el autoconsumo como fin último de la producción campesina sin que esto quiera decir que el sector no utilice como mecanismo de autoabastecimiento productos que no han sufrido ninguna mediación por parte del mercado. Esta constatación autoriza a definir la economía campesina como “parcialmente mercantil" (Tepicht, 1973).

Es la necesidad de comprar los bienes que él mismo no produce la que empuja al campesino a vender parte de su producto y convertirlo así en mercancía. El intercambio de productos en el mercado constituye pues el ámbito característico de la articulación de la economía campesina con el sistema capitalista. Sin embargo las transacciones comerciales, pueden aportar resultados distintos para diferentes grupos campesinos; más que indicar una posible flexibilidad del mercado, los diferentes resultados de la comercialización encuentran su origen en la desigualdad del 
acceso a los medios de producción, en las dificultades mayores o menores que tienen las células familiares para adquirirlos y combinarlos según proporciones técnicamente adecuadas, y en las características del grupo doméstico. (Appendini, Martínez, Rendón y Salles, 1976). Por lo tanto es de la heterogeneidad de los productores originada en condiciones que anteceden el proceso inmediato de producción de donde surgen las diferencias en la retribución del trabajo dedicado a la elaboración de bienes para la venta (Salles, 1981).

De la naturaleza de estas retribuciones -que pueden ser menos o más favorables- dependerán por supuesto las pautas y tendencias del proceso de diferenciación social entre los grupos campesinos.

Para caracterizar las transacciones comerciales de las unidades de producción campesinas, Tepicht (1973) propone la delimitación de dos formas de interacción con el sistema: la compra de medios de producción y la venta de productos. Cuando la venta de productos se hace con mayor intensidad que la compra de medios de producción (Intensidad I), se estimula un mejor aprovechamiento de los recursos y de los bienes de producción generados en el predio campesino. Además, se utilizan como medios intermedios de producción subproductos originados marginalmente en la propia finca o elaborados para el consumo en el proceso de producción.

Cuando predomina la Intensidad I, el renglón compra de medios de producción de origen industrial es débil, lo que de por sí funciona como elemento para definir niveles de capitalización, de autoconsumo intermedio, de diversificación de las relaciones de carácter mercantil, etc. Cuando la compra de medios de producción es importante (Intensidad II), caracteriza procesos sociales en que la economía campesina funciona como mercado para la industria.

Es evidente que, para delimitar ambos tipos de intensidad, hay que reconocer su interdependencia, ya que se refieren a un mismo fenómeno: las formas de intercambio del productor campesino en el mercado. A pesar de que muchas de las reflexiones que se presentan en este texto se refieren al campesino tradicional, no está por demás recalcar que el predominio de la Intensidad II, lleva a la caracterización de un tipo de productor más parecido al farmer. 
A partir del reconocimiento de que el intercambio es una categoría crucial para la definición del campesinado, y de que la comprensión del comportamiento de la unidad de producción pasa obligatoriamente por el estudio de la dinámica de las relaciones entre el campesinado y el sistema, Archetti (1980) enfatiza la necesidad de delimitar los contex tos en que se da la integración de los productores.

En efecto, los campesinos, con el objeto de producir y comerciar, establecen múltiples relaciones personales con diversos agentes que participan en el mercado (prestamistas o instituciones de crédito, comerciantes, etc.), ya que conseguir y renovar préstamos, comprar medios de producción y de vida y vender mercancías son en la actualidad acciones imprescindibles para el funcionamiento económico de estos productores.

No obstante, lo que se llama usualmente mecanismos estructurales de dominación del mercado capitalista sobre los productores campesinos (originados por el funcionamiento del capital sociales y reflejados en los precios de los productos que se venden y se compran, en las tasas de interés y en los niveles de productividad requeridos socialmente para la valorización de las mercancías y del trabajo en ellas contenido), entorpecen la apropiación del remanente del producto creado, una vez deducido lo necesario para la reanudación del proceso de producción y la manutención de la familia campesina. A veces, las restricciones que sufren los campesinos les impiden apropiarse incluso de lo necesario para la reproducción simple del proceso de producción (Lautier, 1979).

La existencia de bajos niveles de productividad del trabajo en el sector campesino, que está en el origen y es un obstáculo para la retención de excedentes, está fuertemente condicionada por el tipo de relaciones que se establecen entre diferentes clases de productores en el mercado. Sin embargo, las modalidades de captación del sobretrabajo campesino dependerán no solamente de la multiplicidad de las relaciones creadas, sino también de su naturaleza, lo que otorga a la red de relaciones establecidas el carácter de espacio necesario para el estudio de la producción campesina (Salles, 1981).

Pero su dificultad para apropiarse del excedente, además de definir la situación de los campesinos en sociedades capitalistas, ha sido una constante histórica. "Igualmente los rasgos esenciales de la producción doméstica no son privativos de la economía 
campesina, los comparten células productivas que constituyen la base de casi todos los modos de producción precapitalistas fundados sobre la agricultura. Vemos pues que se impone considerar en una perspectiva histórica la relación que se establece entre la economía campesina y las sociedades globales" (Appendini, Martínez, Rendón y Salles, 1976).

En efecto, los campesinos han sobrevivido en diferentes formaciones sociales, y su poder de adaptabilidad a ellas los ha hecho interiorizar, recodificándolas en sus propios términos, las leyes económicas de los sistemas dominantes (Tepicht, 1973). Para contrarrestar los condicionamientos desventajosos a que siempre han sido sometidos (el más sobresaliente es la limitación impuesta histórica y estructuralmente para su acceso a la tierra y a los medios de producción), los campesinos han demostrado su capacidad para crear diferentes tipos de respuesta. Con la generalización de nuevas formas de organizar la producción y el trabajo, sancionadas socialmente, y la creación de nuevas necesidades, los campesinos hoy día son empujados a redefinir las condiciones que les permitan asegurar su supervivencia: intensificar el trabajo familiar, diversificar labores, enviar fuerza de trabajo fuera del predio, especializarse en determinadas actividades, son algunos ejemplos. Además, aceptar trabajar por una remuneración muy baja, que evidentemente obliga a la reducción del consumo, es igualmente un aspecto de la adaptabilidad campesina a las imposiciones del sistema. Las respuestas indicadas no son excluyentes y en la realidad de la cédula familiar campesina aparecen combinadas, lo que constituye un rasgo importante de su reproducción.

En efecto, es realizando un complejo conjunto de actividades como las familias campesinas garantizan su continuidad social frente a las tendencias impuestas por los macroprocesos. Siempre que se considera a los campesinos, no como una unidad de producción aislada sino en tanto que sector (en tanto que individuos que, al identificar sus interese sociales comunes, crean relaciones para actuar como fuerza política), se advierte que su comportamiento afecta las determinaciones macrosociales, al crear los espacios para su permanencia: el contrarrestar -o aun impedirel cumplimiento de las tendencias que los empujan a la proletarización es parte de la lucha de los campesinos por la supervivencia. Desde esta perspectiva, la lucha por la supervivencia sobrepasa 
los aspectos económicos más inmediatos vinculados a la organización cíclica de la familia para producir y vender y, así, subsistir; las acciones y reacciones y la adaptabilidad de los campesinos -elementos ligados a la creación de espacios para sobrevivir-los transforma en protagonistas de luchas políticas en aquellas sociedades que ponen límites a su reproducción (Salles, 1981).

Sin embargo, se ha demostrado que el campesinado no ha tenido la posibilidad histórica de cambiar -desde una óptica propia- los rasgos básicos de funcionamiento de las sociedades globales en que ha vivido. A pesar de haber participado en muchas rebeliones locales y hasta en revoluciones (Wolf, 1972), como fuerza principal, no hay actualmente evidencias sobre la factibilidad de implantar una "vía campesina" para el desarrollo global de la sociedad. Pero esto no impide que dejen su huella; en efecto, en los países donde existen campesinos encontramos muestras de su presencia cultural, política y económica.

En México, los campesinos se agrupan físicamente en zonas y comunidades rurales, constituyéndose en ocasiones en el núcleo mismo de pequeños pueblos. En estos espacios los campesinos interactúan con sus iguales, establecen redes de relaciones sociales, culturales, políticas, religiosas, económicas. Lugar privilegiado para las asociaciones locales, estos espacios son también el escenario de luchas contra el poder caciquil y de los conflictos intercampesinos (Santibañez, 1980; Salles, 1981). Cabe señalar, además, que muchas de las contiendas que se dan en el marco de la política nacional, a través de confederaciones, sindicatos y grupos de presión, tuvieron su origen en zonas de asentamientos campesin os (Torregrosa, 1980, Appendini y Salles, 1981).

Estos últimos tipos de acción, al mismo tiempo que indican los aspectos políticos de la lucha de los campesinos por la supervivencia enseñan que su reproducción no se limita a la adaptabilidad (aunque éste sea un rasgo importante para la explicación de los mecanismos que utilizan para sobrevivir). Por lo tanto, el contexto de la reproducción campesina corresponde a un ámbito más amplio, el de las relaciones entre grupos y fuerzas sociales con las cuales interactúa, ámbito que estará en la base misma del concepto de reproducción social (Bourdieu y Passeron, 1981).

El reconocimiento de que la reproducción del campesinado ocurre en diversos ámbitos, permite dar prioridad a niveles de análisis, con el fin de precisar los mecanismos que facilitan, difi- 
cuitan o imposibilitan la supervivencia de este amplio conjunto de individuos.

Trabajo y producción

\section{Condicionantes SOCIALES}

La unidad de producción campesina, al organizarse en torno a 1a familia que busca satisfacer sus necesidades de consumo a través del trabajo realizado con medios de producción propios, parecería ser autocontenida. No obstante, si consideramos las situaciones que son generadas independientemente de la unidad de producción familiar (pero que influyen en ella), pronto nos daremos cuenta de que 1a apreciación de los rasgos invariables de la economía campesina sólo cobra sentido si se tienen en cuenta los vínculos que existen entre su particular modo de operar y el sistema dominante (Archetti, 1978).

La reorientación del patrón de consumo productivo, implícita en la intensificación de las compras de medios de producción y tecnología industrial, interviene en la organización interior de la econom ía campesina en diferentes niveles; señalo el más evidente que es el cambio en la proporción entre el trabajo vivo y los factores de la producción, lo que altera uno de sus rasgos históricos. Entonces, el tránsito entre la Intensidad I y la Intensidad II, además de señalar un cambio interno en la célula familiar de producción, es al mismo tiempo el indicador de una redefinición de la articulación de la economía campesina con el sistema dominante.

Desde este punto de vista, el uso de tecnología en la economía campesina funciona como un indicador de doble alcance: por un lado, caracteriza la heterogeneidad de los productores y de las familias y, por otro, pone en evidencia la existencia de diferentes contextos de integración al mercado capitalista. En efecto, es a partir de este último como se determinarán no sólo los niveles técnicos requeridos para la producción (sea ella campesina o capitalista), sino también los tipos de tecnología que serán utilizados. Por lo tanto, es posible señalar que los cambios internos se realizan por el impacto del sistema que "engloba" la economía campesina, y que sus consecuencias resultan ser la 
contex tualización, a nivel familiar, de situaciones originadas en el ámbito estructural, a través de macroprocesos.

La tierra no escapa a cierto tipo de regulación generada socialmente para toda la agricultura y para todos los productores, aunque la influencia de esa regulación sea distinta en los diferentes sectores sociales del campo. A continuación indico algunas condicionantes de carácter general.

a) La delimitación del espacio reconocido como rural. Este se determina a partir de la división entre el suelo agrícola y el suelo urbano: las proporciones relativas varían según la importancia del crecimiento de la ciudad, lo que representa un tipo especifico de determinación de la tierra disponible para la agricultura. Esta afirmación se constata al estudiar el caso mexicano, en el que, en parte, la actual desapropiación de tierras ejidales (antes alejadas de las zonas urbanas pero hoy día circunvecinas) se hace mediante la presión del crecimiento gigantesco de ciertas ciudades. Regiones que hace un par de décadas podían ser incluidas en la frontera agrícola, con un número considerable de productores campesinos ejidales, ahora contienen espacios conflictivos creados por el crecimiento del área de la ciudad de México. E1 desplazamiento de la frontera agrícola constituye el resultado de este proceso (Schteingart, 1983).

b) E1 carácter imprescindible de la tierra y de sus productos. Aunque el incremento de la productividad del trabajo agrícola haya posibilitado históricamente disminuir tanto el número de personas como las extensiones de tierra que se asignan a la agricultura, no existen evidencias de que la tecnología industrial puede sustituir la tierra para la obtención de determinados productos: así es que aun las industrias instaladas en el campo se ocupan de procesar los productos que se originan en el trabajo desplegado sobre la tierra. A pesar de que en ciertas ramas de la producción agrícola ha ocurrido la sustitución de unos productos por otros que se obtienen industrialmente (algodón por fibras sintéticas, por ejemplo), este proceso se ha mostrado hasta ahora lento y con escasas probabilidades de generalización. Algunas excepciones surgen al observarse la relativa autonomía que adquiere la ganadería a través de modernos métodos de crianza de animales: la ganadería intensiva en tecnología se independiza relativamente de la tierra y se limita a exiguas extensiones (este es el caso de la producción de gallinas). Sin embargo, para ciertas 
actividades agrarias, como por ejemplo la producción de granos -rama en la que existen cultivos intensivos realizados con avances tecnológicos (las semillas mejoradas que proporcionan elevados rendimientos ilustran este fenómeno)-, aún son necesarias grandes extensiones de tierra, lo que otorga a ésta el carácter de imprescindible para la fabricación de una parte no despreciable de mercancías indispensables para la reproducción de la población (alimentos) y de ciertos procesos de producción industrial (materias primas).

c) La imposibilidad de producir la tierra. Dado que la tierra, "condición de producción" agrícola fundamental, no es producto del trabajo humano, cobra de manera natural el carácter de monopolio. Las tierras con una cierta calidad y localización son escasas y su apropiación se transforma inmediatamente en un privilegio excluyente (Marx, 1967).

Lo anterior se aplica a la tierra en general. Cabe presentar otros aspectos que se refieren sobre todo a la tierra campesina. En las sociedades capitalistas, la apropiación de la tierra por los campesinos se ve dificultada por el monopolio "histórico" ejercido por los terratenientes. Sin embargo, el campesinado puede abrir espacios políticos para que la tierra le sea concedida. Es el caso de las reformas agrarias. En el caso de México, marco de referencia de los análisis que presento al final, al predominar un tipo de relación entre los campesinos y el sistema (a través del mercado), que no implica un cambio sustancial en el patrón de consumo productivo, los condicionamientos que se imponen a la economía y la tierra campesina presentan rasgos particulares.

En efecto en México la tierra campesina constituye un bien del que se dispone por el ejercicio de un derecho y como resultado de una lucha (Bartra, Paz, Lanz y Moguel, 1977), sea global y nacional (que cristaliza en la presión que ejerce el campesinado en cuanto fuerza política sobre los grupos que detentan el poder del Estado), sea particular y localizada (que tiene lugar, por ejemplo, en el ámbito de un ejido por el reconocimiento de los derechos de los individuos y las familias que conviven en ese microsistema de poder). No obstante, en el proceso de reforma agraria, al mismo tiempo que se entregan tierras a los campesinos, se crean los mecanismos de su regulación; así es que todos los atributos de la tierra campesina -como calidad, extensión, forma jurídica que adopta la propiedad- han estado íntima- 
mente relacionados con la política del Estado que, influida por diferentes tipos de presión sociopolítica, ha sido cambiante (Salles, 1981).

La función de la tierra campesina entregada por la reforma agraria ha sido objeto de polémicas; inicialmente se concebía al ejido y su explotación como una fuente de ingresos adicionales, para complementar el trabajo que el jornalero desempeñaba en las grandes fincas (Silva Herzog, 1964). A pesar de los cambios jurídicos que se introdujeron en esta concepción, en la práctica el reparto permite al campesinado un acceso a la tierra en condiciones restrictivas. Las reglamentaciones jurídicas determinan igualmente la selección de los individuos que tendrán derecho a la tierra: no todo trabajador desposeído de medios de producción puede tener acceso a la condición de campesino. Este derecho está reservado en general a los trabajadores agrícolas que reúnen las características requeridas por las leyes agrarias.

Un aspecto de esta situación es que lo que da al Estado la posibilidad de reglamentar y controlar la tierra, funciona como condición para que el campesinado tenga acceso a ella, a la vez que la aceptación de las limitaciones y controles es lo que permite al trabajador del campo la posesión de este medio fundamental. No obstante, el control sobre la tierra campesina, sancionado socialmente, refleja antes que nada la legitimación de intereses distintos a los de los campesinos, intereses, aquéllos, que son generados por el enfrentamiento de ideas y fuerzas sociales involucradas en el proceso de reforma agraria.

Por lo tanto, a pesar de que la posesión de la tierra constituye la condición fundamental para el trabajo del campesino, el acceso a ella no está normado por las exigencias del productor directo y su familia. Más bien ocurre lo contrario: es a partir de tierras que, en general, tienen el atributo de ser insuficientes en calidad y extensión, que la familia campesina deberá organizar el trabajo y la producción agrícola.

A este tipo de determinaciones (extensión y calidad) que afectan los resultados del trabajo agrícola, los campesinos responden con el desarrollo de otras actividades o con la venta de la fuerza de trabajo familiar. Estas estrategias, que implican una iniciativa campesina para garantizar su supervivencia, no son incompatibles con otras iniciativas y acciones para reivindicar la ampliación de las tierras, por ejemplo, siempre que existan condi- 
ciones locales para la organización y la asociación solidaria de familias y grupos campesinos. Este tipo de respuesta constituye una dimensión adicional a las iniciativas que toman los campesinos para hacer posible su supervivencia.

\section{Condicionantes Naturales QUE INFLUYEN EN LOS TIEMPOS DE PRODUCCIÓN}

En la agricultura, el proceso de producción abarca la preparación para la siembra, la siembra y la cosecha así como las etapas intermedias de estas labores. Corresponde, pues, al ciclo agrícola. El proceso de trabajo se refiere a todas las labores requeridas en el cuidado del cultivo y que se realizan durante el proceso de producción. Del conjunto de diferentes procesos de trabajo dependerá el buen éxito de la cosecha. Los procesos de trabajo se llevan a cabo durante el ciclo agrícola y, en conjunto, emplean menos tiempo que el exigido por dicho ciclo, y por lo tanto por el proceso de producción.

El proceso de trabajo se caracteriza, entonces, por realizarse en un tiempo relativamente corto y el proceso de producción, por efectuarse en un tiempo largo. Marx (1967) utiliza para esta formulación los términos procesos de trabajo y actos de producción. Esto, que es válido para la agricultura, puede serlo también para la industria.

No obstante, en la agricultura, a diferencia de la industria (en la que eventualmente pueden ser acortados o alargados), estos tiempos están dados en parte por la naturaleza: el tiempo de maduración natural de una planta afecta el momento de la cosecha, los ciclos climáticos -lluvias, por ejemplo-determinan en cierta medida no solamente los periodos de la siembra, sino también las épocas para todos los trabajos que la anteceden.

Los determinantes naturales mencionados influyen tanto en el proceso de producción agrícola, cuyos tiempos además de largos son relativamente incontrolables para el hombre, como en el proceso de trabajo, en que se alternan requerimientos intensivos en labores (periodo de siembra, cosecha, etc.) y aquellos de poca intensidad (entre cosecha, por ejemplo). Esto funciona actualmente para cualquier producción que se realiza con base en la tierra, sea o no campesina. 
Así es que, en la agricultura capitalista, el tiempo de rotación del capital invertido estará influido por los requerimientos naturales impuestos a la duración del proceso de producción. Los volúmenes de capital destinados a pagar salarios varían según las características del proceso de trabajo (como también del tiempo de rotación) y pueden adquirir proporciones importantes sobre todo en las etapas que exigen de labores intensivas.

Este mismo tipo de razonamiento puede ser aplicado a la economía campesina; el dinero utilizado en la compra de insumos y de mercancías destinadas al consumo familiar es adelantado por el tiempo del proceso de producción, y los requerimientos de labor intensiva de ciertas etapas del proceso de trabajo exigirán igualmente sumas de dinero adicionales para pagar salarios, siempre y cuando la fuerza de trabajo familiar no sea suficiente $y$ se necesite contratar trabajo ajeno; tales sumas tardarán en refluir al campesino el tiempo exigido por el proceso de producción.

Sin embargo, la diferencia entre tiempo corto (proceso de trabajo) y tiempo largo (proceso de producción) provoca efectos distintos en las formas de producción que utilizan exclusivamente trabajo asalariado (capitalista) y las que se apoyan principalmente en el trabajo familiar. Señalo apenas los efectos sobre la organización del trabajo, a pesar de que el desfasamiento entre estos dos tiempos introduzca una muy amplia gama de consecuencias para la producción en la agricultura.

Las empresas capitalistas contratan el volumen de fuerza de trabajo requerido por el desarrollo de la producción y tienen que cuidar que la proporción de trabajo sea adecuada a los medios de producción disponibles. La eficiencia de la empresa será tanto mayor cuanto más se acerque a una cierta combinación entre fuerza de trabajo y medios de producción durante los procesos de trabajo, y que deberá de enmarcarse en el monto total del capital disponible.

En tales empresas, existe la posibilidad de regular la fuerza de trabajo según las condiciones impuestas por los ciclos naturales a la producción agrícola. La existencia de fuerza de trabajo móvil es indispensable para ciertos cultivos en determinadas etapas: la pisca del algodón o el corte de la caña, por ejemplo, requieren de grandes contingentes de trabajadores por lapsos muy cortos. A pesar de las dificultades que se presentan para la 
contratación y posterior despido de la fuerza de trabajo eventual, la existencia de ésta da flexibilidad a la organización de la empresa y más, aún, permite contrarrestar los efectos negativos de los requerimientos discontinuos en trabajo, característicos del desfasamiento entrè los procesos de trabajo y de producción.

A diferencia de los capitalistas que empiezan a producir comprando la fuerza de trabajo necesaria, los campesinos organizan la producción a partir de un factor ya dado que es la fuerza de trabajo familiar. "Las relaciones que se establecen entre los miembros de la familia sobre la base de lazos de parentesco activan principios de interdependencia y solidaridad familiar alrededor de las acciones necesarias para su sobrevivencia. Esta tiene por condición concreta la común explotación del patrimonio familiar" (Martínez y Rendón, 1981).

Si consideramos la hipótesis de que el trabajo realizado sobre la tierra (patrimonio fundamental de la familia campesina) es una actividad importante para la reproducción del grupo doméstico, y que, por esta misma razón, funciona como eje articulador de las otras labores llevadas a cabo por la familia, se observa la siguiente situación: los requerimientos de trabajo discontinuo que caracterizan la producción en el campo e imponen un ritmo particular a la organización de las actividades agrícolas, crean loś espacios para la diversificación de las labores. Estas son complementarias del trabajo especificamente agrícola y contrarrestan la propensión a la subutilización de la mano de obra familiar, originada por el'carácter discontinuo del trabajo en la producción agrícola. Es cierto también que esta discontinuidad estorba las actividades extraagrícolas del campesino, al introducir una segmentación de las mismas.

La carencia de recursos, como el riego, por ejemplo, impide una explotación más prolongada y continua de la tierra. En terrenos de temporal, a veces sólo es posible una siembra, que coincide con el periodo de lluvias; como resultado, la "desocupación", además de estar presente en las etapas en que el proceso de producción se desarrolla sin requerir de labores, se extiende a la temporada de sequía, en que la agricultura es impracticable para los campesinos por la ausencia de un tipo específico de tecnología: el riego.

Cabe, por lo tanto, la consideración de que los espacios para la realización de otras labores se crean, naturalmente, por los 
ciclos agrícolas que condicionan los montos de trabajo requeridos en la agricultura y, socialmente, por la escasez de recursos, medios de producción y tecnología, que permitan una mayor continuidad del trabajo agrícola.

En efecto, para el caso mexicano ya se constató, a través de numerosas investigaciones realizadas en zonas y comunidades campesinas, que el trabajo agrícola no siempre permite la generación de los ingresos necesarios para la reproducción de la unidad doméstica (Díaz Polanco, 1976). Sea para dar continuidad a la producción agrícola, sea para el consumo personal, la dispersión productiva se presenta como condición indispensable para la supervivencia del núcleo doméstico, en importantes sectores del campesinado.

Las combinaciones del trabajo agrícola con otras actividades son numerosas. Varían desde la situación en que los espacios para las demás labores se fijan a partir del trabajo agrícola, hasta el extremo opuesto, en que la tierra es utilizada como simple asentamiento para campesinos artesanos o para proletarios. No obstante, cuando hay actividad agrícola, aun en proporciones pequeñas, ella está determinada por los factores naturales que a su vez determinan la duración de los procesos de trabajo y de producción.

En el caso de que la producción agrícola pueda ser ampliada o intensificada, el núcleo familiar tenderá a la especialización en una o varias líneas de productos. La pecuaria -entendida como actividad principal y no subsidiaria-puede ser transformada en factor de especialización de la producción, siempre y cuando el núcleo campesino disponga de la tierra y de los recursos requeridos para esta labor. En su carácter de producción principal normaría los tiempos para la agricultura y otras labores.

\section{La formación del ingreso global}

El uso de trabajo vivo

La familia campesina, al organizar la producción a partir de ciertos elementos prefijados social y naturalmente (hemos visto con algún detalle solamente la tierra), intenta optimizar al trabajo familiar que se aplica a los recursos disponibles. 
La necesidad de funcionar productivamente con medios de producción restringidos es la razón por la cual, en la economía campesina, la actividad agrícola se hace con el predominio del trabajo vivo sobre los demás factores de la producción, que regulan su uso (Bartra, Paz, Lanz y Moguel, 1976).

Una vez que en otros sectores de la producción, e incluso en el campo, se generaliza el trabajo realizado por medio de la máquina y de la tecnología moderna (fertilizantes, semillas mejoradas, riego, etc.), y que el campesinado no tiene posibilidad de disminuir la proporción de trabajo vivo, se presentan consecuencias nefastas para este sector, aunque éstas sean distintas para los diferentes grupos que lo conforman. La escasa productividad del trabajo es una de estas consecuencias y tal vez la más grave: al significar una relación muy baja entre unidad de producto y unidad de trabajo, indica que los productos campesinos están preñados de elevados - aunque variados- niveles de trabajo. En estas condiciones, una mercancía campesina, por ejemplo el maíz, al competir con la misma mercancía pero obtenida con más altos niveles de productividad, difícilmente conseguirá la retribución de todo el trabajo desplegado cn su producción, una vez que se venda al precio de mercado, que es formado según la productividad del trabajo y de la tierra del sector capitalista. Una explicación posible aunque simplificada para esta situación, es que si la sociedad sancionara positivamente, a través de precios, todo el trabajo contenido en el producto campesmo, éste resultaría socialmente muy caro.

La desvalorización de las mercancías campesinas que concurren al mercado significa una desvalorización del trabajo que las produjo. Este fenómeno, al servir de base para la explicación de los bajos niveles de ingreso, sirve también para la comprensión del proceso de pauperización de los productores campesinos. Desde esta perspectiva, las condiciones del campesino se vinculan intimamente con las determinaciones del mercado capitalista que, obedeciendo a una lógica estructural, castiga a los productores que no consiguen una relación adecuada entre trabajo y medios de producción.

Volveremos, pues, a la idea de que las condiciones sociales que anteceden al proceso de producción (además de funcionar como variables para describir la heterogeneidad campesina), crean, a su vez, las condiciones para competir en el mercado. El 
mercado funciona, así, como una instancia que impide la adecuada valorización del trabajo campesino, al imponer la de las mercancías a partir de niveles de productividad que no son propios del campesinado (Margulis, 1979). Por esta razón, los campesinos logran retirar del valor total creado socialmente apenas una fracción, que en ocasiones no llega a cubrir la totalidad del "trabajo necesario".

Conviene, sin embargo, no perder de vista que la tendencia a utilizar una elevada proporción de trabajo vivo en relación a otros medios de producción, portadores de trabajo cristalizado, disminuiría en la medida en que la vinculación del campesino con el mercado se acercara a la Intensidad II, lo que de por sí evoca el carácter variable de esta situación, y al mismo tiempo invita a una flexibilidad analítica para captar la situación campesina desde una perspectiva dinámica. En efecto, "en tal o cual formación social el aparato de gobierno se podrá ver en la necesidad de dictar medidas extraeconómicas (precios oficiales, subvenciones, etc.) que alteren las leyes del mercado, debido a la importancia numérica del sector campesino, al peso que tienen en el mercado interno como parte de la oferta o de la demanda de determinados bienes, o a la fuerza social y política que representa. Sin embargo se tratará solamente de modificaciones coyunturales a la estructura de precios" (Appendini, Martínez, Rendón y Salles, 1976). Pero, seguramente, mecanismos de esta naturaleza amplían los espacios para la reproducción campesina. Reflejan también un tipo de interacción de la clase campesina con las fuerzas sociales ubicadas en el aparato de Estado, que tiene como resultado la creación de coyunturas favorables para los campesinos.

Si regresamos al ámbito micro de la célula familiar campesina, observaremos que otras labores se realizan igualmente mediante un uso intensivo de trabajo vivo. En efecto, en el campo, la producción de los medios de vida y la ejecución del quehacer doméstico exigen mayor trabajo a las familias, en comparación con otros sectores de la sociedad. A pesar de que la tecnología en algunos contex tos hay a acortado sustancialmente los tiempos dedicad os a las labores del hogar, la familia campesina aún tiene que hacer mucho esfuerzo para llevarlas a cabo.

La elaboración de tortillas, por ejemplo, se realiza en determinadas situaciones con poca inversión de trabajo, porque está 
mediada por una cierta tecnología (molinos de nixtamal, máquinas tortilladoras, etc.). Cuando la familia campesina no goza de estas ventajas, lo que es frecuente, se alarga desmesuradamente el tiempo que hay que invertir en su elaboración, que varía según el número $\mathrm{y}$ habilidades de las personas dedicadas a ello, según los patrones culturales locales, etc. Aún así, la preparación de tortillas, desde la molienda hasta el producto final, para el consumo diario de una familia de tamaño medio, requiere de varias horas de trabajo femenino (Young, 1978). De todos modos, la familia campesina realizará esta labor y las demás que sean necesarias para la reproducción del núcleo familiar, por costosas, en cuanto a trabajo, que sean.

En estos casos existe igualmente una suerte de desvalorización del trabajo familiar campesino, que por supuesto no se manifiesta de manera necesaria a través de los precios, pues los productos de trabajo doméstico, en general, son consumidos por la familia sin ninguna mediación del mercado. La "desvalorización" consiste en la ausencia de una remuneración para los servicios y productos que de todos modos han costado trabajo. La forma en que las estadísticas agrícolas captan esta situación, a través de la variable "familiares no remunerados que trabajan en el predio", de hecho rinde cuenta de una realidad empírica muy importante. Teóricamente se puede argüir que la función del trabajo doméstico es la autosatisfacción de las necesidades de los agentes que lo ejecutan, y que por esta misma razón la utilidad que tiene para el consumo familiar es la que funciona como "remuneración" (Salles, 1981). Sin embargo, estos razonamientos esconden mecanismos de explotación más precisos y que se conceptúan como "autoexplotación de la fuerza de trabajo", porque la estructura familiar misma es el agente de este proceso.

El trabajo agrícola

Las características físicas de la tierra, extensión y calidad, influyen decisivamente en la capacidad productiva del trabajo agrícola, ya que determinan directamente los rendimientos. Esta determinación será tanto mayor cuanto menor sea la utilización de medios de producción y de tecnología. Esto funciona para toda la producción agrícola. 
La investigación sobre la cuestión campesina ha demostrado ampliamente que entre estos productores predominan las parcelas pequeñas. Los elementos que contrarrestan las limitaciones en cuanto a la extensión del factor tierra -o sea la tecnología en sus diferentes aspectos-, al permitir montos de productos razonables, no siempre están al alcance de los campesinos. En parcelas pequeñas en ocasiones es imposible sustituir los instrumentos de trabajo tradicionales, aun cuando exista la posibilidad económica de adquirir medios de producción modernos (Boserup, 1979). En estos casos un obstáculo lo es la inadecuación de la tecnología, que se crea sin tener en cuenta los requerimientos de las parcelas pequeñas, y que transforma su adquisición en una inversión riesgosa o antieconómica. Es bien evidente que estos efectos se matizan si hay la disposición y los medios para la asociación de pequeñas parcelas, o si la extensión y localización geográfica de los terrenos individuales presentan condiciones de redituabilidad para el uso de máquinas, lo que suele acontecer en algunas regiones, no así en zonas de minifundismo (Yunes, 1977).

Sin embargo, hay que recordar que todo proceso de adquisición y renovación de medios de producción en la economía campesina (consumo productivo) se relaciona de una manera muy estrecha con la satisfacción de la propia demanda del grupo doméstico, ya que su costo afecta directamente el presupuesto familiar: a menor nivel de bienestar, menores serán pues las posibilidades de inversión, por la necesidad en que se encuentra el grupo doméstico de garantizar primero su consumo inmediato (Appendini, Martínez, Rendón y Salles, 1976).

Si bien es verdad que los requerimientos más amplios del consumo familiar están influidos por pautas culturales diversificadoras y por el patrón de consumo del contexto local en que se ubica la familia campesina, el consumo inmediato (alimentación, vestido, salud, etc.) asume la forma de necesidad básica que deberá de ser satisfecha; en ocasiones, y sobre todo en las familias más pobres, la resolución de los problemás básicos se hace en detrimento de la reproducción del ciclo productivo agrícola.

La "inversión" del tiempo de trabajo familiar en actividades no agrícolas en general ocasionada por la exigüidad de la parcela, o por la imposibilidad de proveerse de los medios de producción 
necesarios, permite a la familia campesina la obtención de un ingreso global de subsistencia, sin haber resuelto el problema de la improductividad del trabajo agrícola.

Las pautas de consumo personal restringido, determinadas por la desvalorización del trabajo campesino (generada socialmente), a la que se suma la frecuente imposibilidad de mantener el proceso de producción sobre la base de elevadas compras de insumos y medios de trabajo, determinan que los costos de producción en la economía campesina sean en general bajos; pero la contrapartida del forzoso ahorro en costos, como se ilustra más adelante con el análisis de los cuadros, es la baja productividad del trabajo agrícola, que sólo posibilita la obtención de escasos rendimientos.

Además de la extensión, otro atributo de la tierra que influye en la productividad del trabajo agrícola, es la calidad. En tanto que elemento crucial para la obtención de buenos rendimientos, la calidad de la tierra funciona indiscriminadamente para todos los productores, sean ellos capitalistas o campesinos. No obstante, es un hecho verificado empíricamente que en México los campesinos en general se ubican en los peores terrenos. Los elementos contrarrestantes de la calidad natural de la tierra -como el riego, por ejemplo-, reducen su impacto negativo sobre los rendimientos, y el incremento de la productividad del trabajo en tierras irrigadas es indiscutible, como veremos a continuación. Sin embargo, aunque existan tierras ejidales con riego, importantes sectores de los campesinos no cuentan con este recurso.

La ausencia de riego, en efecto, imposibilita el cultivo de algunos productos (algodón), provoca rendimientos notablemente inferiores en ciertos granos que igualmente pueden ser producidos en tierras de temporal (maíz) y, además, excluye una posible flexibilidad en el calendario agrícola, pues la siembra en tierras de temporal depende del periodo de lluvias. La consecuencia es que la producción se realiza sobre todo en el ciclo agrícola de primavera-verano quedando excluido del calendario agrícola el ciclo de otoño-invierno para los campesinos que trabajan tierras de mal temporal en regiones poco húmedas.

Los datos presentados en el cuadro 1 ejemplifican algunas de las situaciones indicadas, pues se refieren a los rendimientos en producto (maíz) que se obtienen cuando varían los montos 
de los gastos aplicados a iguales unidades de tierra (hectárea), que sin embargo tienen calidades distintas (mal temporal y buen temporal). Los datos fueron obtenidos por la Dirección General de Economía Agrícola (DGEA) a través de la aplicación de 5000 cuestionarios en los 17 estados más importantes en cuanto a la producción de maíz, que aportaron el 85 por ciento del total nacional de este cultivo (Dirección General de Economía AgrícolaSecretaría de Agricultura y Recursos Hidráulicos, 1978). Se establecieron tres calidades de tierra de temporal. El cuadro que analizo excluye el tercer tipo (muy buen temporal) porque los datos no abarcaban todas las categorías de gastos en insumos y servicios.

Es obvio que los rendimientos de maíz por hectáreas varían en función de la calidad de la tierra y del monto de gastos en insumos y servicios, sin embargo, es interesante observar más detenidamente algunas relaciones que se derivan de este marco general.

a) Si tomamos los casos extremos, en los que hay variaciones entre tipos de tierra y entre gastos en insumos y servicios, se observa que en la tierra $A$ con bajos gastos y en la tierra $B$ con altos gastos, los rendimientos en producto (maíz) son de 495 y 1873 kilogramos por hectárea respectivamente. El resultado es que los rendimientos de la tierra $B$ son aproximadamente cuatro veces superiores a los de la tierra $A$ (o sea que la diferencia es de 280 por ciento). Este ejemplo, además de indicar que la heterogeneidad entre productores agrícolas se basa en la desigualdad de recursos, ilustra la profundidad de la misma. En el supuesto de que toda la producción se vendiera (lo que es una hipótesis, porque en efecto el autoconsumo del maíz producido en tierras temporales es elevado) a los precios de garantía vigente, que para el periodo eran de 2340 pesos por toneladas, los productores, en las oeores condiciones de la tierra $A$, obtendrían un ingreso bruto por hectárea de 1130 pesos aproximadamente, mientras que los productores de la tierra $B$ alcanzarían un total de 4210 pesos.

b) Cuando mejora la calidad de la tierra, los incrementos en los rendimientos del maíz son notables, especialmente si se cuenta con el nivel medio en gastos. Los rendimientos varian de 646 a 1172 kilogramos por hectárea (lo que representa un incremento de 81 por ciento). Por lo tanto, en este caso, un mayor gasto en 
insumos funciona como un "efecto multiplicador" de la calidad de la tierra.

c) Para un mismo tipo de tierra (la $A$, por ejemplo) se observa un fuerte incremento en los rendimientos cuando los gastos en insumos y servicios se elevan del nivel bajo al medio ( 30 por ciento). Sin embargo, este mismo tipo de tierra $(A)$ presenta incrementos muy superiores (63 por ciento), cuando los gastos se elevan del nivel medio al alto. Este resultado nos indica que es solamente a partir de un cierto monto de gastos que se logran rápidos incrementos en los rendimientos en las tierras de peor calidad (mal temporal). Esta evidencia ilustra, en parte, las dificultades que enfrentan los campesinos con muy escasos recursos para llegar a los niveles de gastos que permitan una combinación técnica adecuada para lograr mejores rendimientos.

d) La tierra $B$ presenta una notable mejoría en los rendimientos cuando se elevan los montos de los gastos; en efecto, los volúmenes de producto son 715, 1172 y 1873 kilogramos por hectárea, respectivamente, para los tres niveles de gastos; sin embargo, sus tasas de incremento son aproximadamente las mismas, o sea de 64 y 60 por ciento, lo que indica que, cuando la calidad de la tierra es mejor (buen temporal), los aumentos en rendimientos se hacen notar muy rápidamente y se presentan desde el primer paso en otra categoría de gastos en insumos y servicios.

e) Las dos hipótesis mencionadas en los incisos $c$ y $d$ parecen confirmarse si se observan los rendimientos en la tierra $A$ y en la $B$ con gastos en insumos que varían del primer al segundo nivel $y$ del segundo al tercer nivel respectivamente; en efecto, los rendimientos se incrementan en los primeros casos (44 a 81 por ciento) y se mantienen aproximadamente constantes en los segundos ( 81 a 78 por ciento).

$\mathrm{Al}$ analizar los datos del cuadro 2, que arrojan más elementos sobre las condiciones técnicas, se observa que la utilización de medios de producción diferentes (yuntas o máquinas) y de tierras de calidad distinta (riego o temporal), provoca diferencias notables en los rendimientos obtenidos. A diferencia del cuadro anterior, las tierras de temporal se presentan como una categoría homogénea, lo que impide la comparación de los dos cuadros. La introducción de máquinas, aunada a un alto uso de insumos $\mathrm{y}$ servicios, en procesos de producción que se realizan en las 
Cuadro 1. Rendimiento del maí $(\mathrm{Kg} / \mathrm{Ha})$, según calidad de la tierra y gastos en insumos y servicios

\section{Gastos en insumos y servicios (pesos $/ \mathrm{Ha}$ )}

\begin{tabular}{lccccc}
$\begin{array}{l}\text { Calidad de } \\
\text { la tierra }\end{array}$ & $\begin{array}{c}\text { (bajo) } \\
\text { hasta 100 } \\
(1)\end{array}$ & $\begin{array}{c}\text { incremento de } 100 \text { a } 700 \\
\text { entre 1 y } 2\end{array}$ & $\begin{array}{c}\text { (2) } \\
\text { incremento } \\
\text { entre 2 y 3 }\end{array}$ & $\begin{array}{c}\text { (alto) } \\
\text { (3) }\end{array}$ \\
$\begin{array}{l}\text { Mal tem- } \\
\text { poral (A) }\end{array}$ & 495 & $30 \%$ & 646 & $63 \%$ & 1054 \\
$\begin{array}{l}\text { incremento } \\
\text { entre A y B }\end{array}$ & $44 \%$ & $81 \%$ & & $78 \%$ \\
$\begin{array}{l}\text { Buen tem- } \\
\text { poral (B) }\end{array}$ & 715 & $64 \%$ & 1172 & $60 \%$ & 1873 \\
\hline
\end{tabular}

Resumen del Cuadro VIl elaborado por DGEA-SARH, en Análisis económico del cul. tivo del maíz en México en el ciclo primavera-verano.

Fuente: Encuesta primavera-verano, 1975 (DGEA-SARH).

Cuadro 2. Rendimiento del maíz $(\mathrm{Kg} / \mathrm{Ha})$, según tecnología (Ciclo primavera-verano)

Combinación tecnológica
$\begin{gathered}\text { Rendimiento } \\ (\mathrm{Kg} / \mathrm{Ha})\end{gathered}$

(1) Temporal, yunta y bajo uso de 540 insumos y servicios

(2) Temporal, yunta y uso medio $\quad 1000$ de in sumos y servicios

(3) Temporal, yunta y alto uso de 1349 insu mos y servicios

(4) Temporal, maquinaria y alto $\quad-1733$ uso de insumos y servicios

(5) Riego, maquinaria y alto uso de insumos y servicios

Resumen del cuadro elaborado por DGEA, op cit., p. 10.

Fuente: DGEA-SARH, encuesta primavera-verano, 1975. 
tierras de riego, permite la obtención de elevados rendimientos en producto (3 375 kilogramos por hectáreas). Si se comparan estos resultados con los 540 que se obtienen en la categoría (1), se ponen de evidencia las desventajas del productor campesino más pobre, que trabaja con yuntas, en mal temporal y con bajo uso de insumos o servicios. Se ilustra también la productividad extremadamente desigual del trabajo realizado por medio de máquinas y por medio de yuntas, sobre todo si se toma en consideración los casos extremos, que seguramente son ocupados por campesinos pobres y por productores capitalistas ( 1 y 5 ). Pero si se observan los rendimientos en las dos últimas categorías (4 y 5), ambas con maquinaria y alto uso de insumos y servicios, se puede concluir también que la calidad de la tierra (riego) juega un papel muy importante en el incremento de la productividad del trabajo, que se refleja en montos de productos muy desiguales ( 3575 kilogramos por hectáreas en riego y 1733 en temporal).

Entre tanto, al comparar las progresiones de los rendimientos de las categorías 1 y 4 por una parte (en que los rendimientos suben en proporción de 1 a 3 aproximadamente), y las categorías 4 y 5 por otra (en que los rendimientos suben en proporción de 1 a 2 aproximadamente), concluimos que la elevada tecnología produce un efecto más notable que la calidad de la tierra.

Claro está que lo anterior no autoriza interpretaciones globales sobre el campesinado: al indicar el desempeño del trabajo específicamente agrícola, estos datos ilustran heterogeneidades en su resultado. Es solamente a partir del estudio del conjunto de la "actividad económica" de las familias campesinas (diversificación de labores, trabajo fuera del predio, etc.) que se puede apreciar la importancia mayor o menor del trabajo agrícola en la formación del ingreso global y, por lo tanto, el efecto que la baja productividad ocasiona en la reproducción de los productores campesinos.

\section{LA DIVERSIFICACIÓN DE LABORES}

"Lo que empuja a las familias a diversificar e intensificar su trabajo es su incapacidad para asegurar su reproducción global merced a la sola actividad agrícola; pero una vez reconocida 
esta tendencia general se descubre que lo que determina de manera más directa a qué actividad o actividades particulares se consagrará el grupo doméstico es la magnitud y la composición de su fuerza de trabajo" (Martínez, 1980).

Evidentemente esta constatación señala la dificultad que hay al emprender el análisis del fenómeno de la diversificación de labores en las familias campesinas. La morfología de la familia, su tamaño, la edad de los miembros, y los tipos de actividad para los que son aptos constituyen los elementos inmediatos que determinan la organización del trabajo y la selección de labores; los patrones culturales locales, la especificidad de los mercados en que colocarán los productos resultantes de las actividades particulares, funcionan igualmente como un marco obligado para la planeación del trabajo familiar. Sin embargo, el objetivo de la diversificación de labores es el mismo para las diferentes familias: la obtención de un ingreso global que garantice la supervivencia del núcleo doméstico.

El ingreso global deberá de hacer posible la reposición de la fuerza de trabajo y de las condiciones de producción, lo que, además de constituir un rasgo definitorio de la producción familiar campesina, caracteriza todo proceso de reproducción simple de mercancías (Marx, 1967).

Debido a la "dispersión" productiva que implica un patrón de reproducción basado en la diversificación de labores, la reposición de las condiciones de producción requiere de "insumos" de diversa naturaleza, lo que provoca una multiplicación de esfuerzos para proveerse de "insumos" diversificados.

La actividad económica global, que abarca todas las actividades realizadas por la familia campesina (prestación de servicios o elaboración de productos para el mantenimiento directo de la unidad familiar de producción y consumo, o producción de mercancías para la venta), constituye el ámbito analítico mínimo del estudio de la organización del trabajo del grupo doméstico. En contextos como el que estamos analizando, en que la especialización agrícola no es una constante, toda unidàd de análisis basada en la observación de actividades aisladas, al amputar la dimensión de relación entre ellas, indica apenas aspectos parciales de la reproducción familiar campesina. En consecuencia, es a partir del estudio de la formación del ingreso global (tomado como resultado del conjunto de labores que lleva a cabo una 
familia con características dadas) como mejor se observa su patrón de reproducción.

Al 13 ?

En la formación del ingreso global interviene un conjunto de labores que no pueden ser cuantificadas en términos monetarios (a pesar de significar un gasto en trabajo) porque no hay la mediación del mercado; además, los individuos que las ejecutan no siempre reciben una remuneración en efectivo, ya que los miembros de la familia funcionan al mismo tiempo como agentes de la producción y como consumidores. A manera de ejemplo podemos mencionar todas las labores relacionadas con la familia (cuidado de hijos y ancianos, preparación de comidas, etcétera), aquellas que sirven de apoyo inmediato al trabajo agrícola (elaboración de alimentos para los que trabajan en la milpa, transportación de los mismos cuando casa y milpa no están integradas en el mismo espacio), atención de hortalizas y ganado menor, y recolección para el consumo final de la familia. Estas labores entrarán en la formación del ingreso global.

a Además, debido a que las unidades de producción campesinas son "parcialmente mercantiles", partes variables de los productos del trabajo agrícola (por ejemplo, el maíz y el frijol) pueden ser autoconsumidos, sea para el mantenimiento de la fuerza de trabajo familiar, sea para el reinicio de un nuevo ciclo productivo. En estos casos la producción agrícola cumple una función, la de satisfacer las necesidades de la unidad de producción y consumo, sin generar un ingreso monetario.

3. La formación dèl ingreso global incluye igualmente las labores cuy os resultados cristalizan en mercancías para el mercado y

a) que, por lo tanto, sólo entrarán en el renglón del consumo familiar o productivo bajo la forma de ingresos monetarios, provenientes de su comercialización (algunos ejemplos son los productos agrícolas, distintos tipos de artesanía - barro, madera, bordados, etcétera-, productos pecuarios y sus derivados -leche, huevos, oueso, etc.- que pudieron incluso haber sido elaborados para el consumo de la familia, pero que por una razón u otra son vendidos). La venta de fuerza de trabajo familiar entrará en la formación del ingreso global siempre y cuando los salarios por ella generados sirvan de apoyo para la reprodueeión de la unidad de producción doméstica.

Estas labores tienden a ser evaluadas y comparadas entre sí: la inversión en tiempo que exigen, los resultados que de ellas se 
obtienen, las aptitudes del grupo familiar para desempeñarlas, componen los criterios para la elección de dichas labores, que se realizarán en función de su factibilidad en el mercado, así como del ingreso que aportarán. Sin embargo, como la evaluación externa de los productos campesinos se hace bajo mecanismos impuestos por las leyes del mercado, sus resultados en términos de ingreso serán variables y en cierta medida imprevisibles, sobre todo si sé trata de bienes no regulados por los precios políticos (de garantía).

A pesar de que apenas algunas de las actividades mencionadas pueden ser cuantificadas en términos monetarios, es a partir del conjunto de ellas que se generará lo que Chayanov (1974) llama el ingreso global indivisible; y es el monto de este ingreso global el que determinará para la familia campesina la pauta de consumo en sus diferentes renglones.

Supongamos, no obstante, que exista una relación inadecuada entre monto de trabajo disponible y medios de producción y otras labores, que ocasione un desfasamiento (descompensación) entre la capacidad de trabajo y el trabajo realmente ejercido (sea debido a la escasez de medios de producción, sea por la incapacidad para ejercer la artesanía u otras actividades, sea aún por la imposibilidad de créditos para una etapa que requiere de gastos).

En estos casos pueden surgir por lo menos dos situaciones: a) la unidad de producción campesina de todos modos mantiene los efectivos de fuerza de trabajo subempleados, sin trabajo productivo o con rendimientos insuficientes, lo que hace que bajen los niveles de consumo; y $b$ ) la unidad de producción campesina envía a sus miembros a trabajar fuera del predio, lo que sin embargo no puede ser tomado exclusivamente como el indicador de una inadecuación de los elementos señalados; puede, entre otras cosas, deberse a la necesidad de incrementar ingresos en dinero.

A modo de conclusión quiero subrayar que la diversificación de labores (que persigue un ingreso global), cuandó implica la necesidad de poner partes variables de la fuerza de trabajo familiar a disposición del capital, da cabida a una forma adicional de vinculación de la economía campesina con el sistema capitalista. Pero esta vinculación adicional -por no tratarse de trabajadores libres y sí de individuos que mantienen aún una relación de pro- 
piedad o posesión con la tierra - puede traducirse en una doble explotación, debido a su inserción en el mercado en tanto que productores pauperizados y en tanto que vendedores de fuerza de trabajo. En efecto, con frecuencia el campesino pequeño productor (o miembros de su familia que aún están atados económica y socialmente al grupo doméstico y al contexto rural del que proviene) vende su fuerza de trabajo por un salario inferior a su costo de reproducción, salario que complementa con el producto de la parcela (Meillassoux, 1977).

Siempre que la familia campesina obtenga un ingreso que le permite reproducirse como unidad de producción, un ingreso mediante la combinación de actividades productivas (en el predio y asalariadas), se podrá hablar de pauperización y de semiproletarización del campesinado. El concepto de proletarización está reservado para las situaciones en que las células campesinas sufren una suerte de degradación de sus condiciones de producción, que no les permiten un "equilibrio económico" a través de la combinación de labores y antes al contrario provocan la liberación del conjunto de la fuerza de trabajo familiar, el rompimiento de vínculos con la tierra y la consecuente pérdida de sus raíces rurales.

En tales situaciones, la formación del ingreso global de supervivencia implicará el desempeño de actividades que, por excluir el trabajo sobre la tierra con medios de producción propios, determinan nuevas formas de vida y nuevos tipos de inserción social, específicos de los trabajadores, que al ser expulsados del campo, se liberan de su condición de campesinos.

\section{Bibliografía}

Appendini, Kirsten; Martínez, Marielle; Rendón, Teresa; Salles, VANI A, "La economía campesina: consideraciones teóricas y análisis preliminar de una encuesta en 13 pueblos" (ed. mimeografiada), CES, El Colegio de México, México, 1976.

ARCHETT I, EduARdo, "Una revisión general de los estudios sobre el campesinado", Estudios Rurales Latinoamericanos, Vol. I, enero-abril, Bogotá, 1978. 
Archetti, Eduardo, "The Growth of Capitalism and Peasant Economy: Some Problems of Transference of Surplus" (ed. mimeografiada), Institute of Social Anthropology, University of Oslo, 1980.

ARIZPE, LOURDEs, "La migración por relevos y la reproducción social del campesinado", Cuadernos del CES, núm. 28, El Colegio de México, México, 1980.

Bartra, Armando; Paz, Lorena; Lanz, Miguel; Moguel, Julio, "La explotación del campesinado en el capitalismo" (ed. mimeografiada), México, 1977.

Boserup, Esther, "El impacto del crecimiento de la población en la producción agrícola", en Urquidi, Víctor y Morelos, José (comps.) Crecimiento de la población y cambio agrario, El Colegio de México, México, 1979

Bourdieu, Pierre y Passeron, Jean Claude, la Reproducción. Ed. Laya. Barcelona, 1981.

Chay anov, AleX ANDer, la organización de la unidad económica campesina, Ed. Nueva Visión, Buenos Aires, Argentina, 1974.

Dirección General de Economía Agrícola de la Slcretaría de Agricultura y Recursos Hidráulicos (SARH), Análisis Económico del cultivo del maiz en México, en el ciclo de primaveraverano, Ed. DGEA-SARH, México, 1977.

Díaz-Polanco, HÉctor, "La economía campesina y el impacto capitalista. Un caso mexicano" en Rodolfo Stavenhagen et al, Capitalismo y campesinado en México, SEP-INAH, México, 1976.

LAUTIER, Bruno, "La subsunción formal del trabajo al capital" en Estudios Sociales Centroamericanos, enero-abril, año 5, núm. 13, San José, Costa Rica, 1976.

MARg Ulis, MARIo, Contradicciones en la estructura agraria y transferencia de valor. Serie Jornadas, núm. 90, El Colegio de México, México, 1979.

Martínez, Marielle y Rendón, Teresa, "Estrategias de reproducción de las unidades domésticas" (ed. mimeografiada), CES, El Colegio de México, México, 1981.

- "Reflexiones a partir de una investigación sobre grupos domésticos campesinos y su estrategia de reproducción" (ed. mimeografiada), CES, El Colegio de México, México, 1982.

Martínez, Marielle, "Comunidad y familia en la dinámica social campesina", Nueva Antropología, año IV, núm. 13-14, México, 1980.

MarX, KARL, Le capital, libro I, tomo 1, Ed. Sociales, París, 1967.

MeIllassoux, Claude, Mujeres, graneros y capitales, Siglo XXI Editores, MéXico, 1977.

Rober tS, Bryan, Cities of Peasants, Edward Arnold Ed., Londres, 1978.

SAlles, VANIA, "Los campesinos: espacios y luchas" (ed. mimeografiada) 
Center for Latin American Studies, Universidad de Florida, Gainesville, 1981.

SANTIBAÑEZ, JUAN JOSÉ, "Los campesinos de un ejido cañero en Oaxaca" (ed.mimeografiada), CES, El Colegio de México, México, 1980.

SCHTEINGART, MARTHA, "La incorporación de la tierra rural de propiedad social a la.lógica capitalista del desarrollo urbano, el caso de México", en La tierra como recurso estratégico para el desarrollo y la transformación social, Ed. SIAP, México, 1983.

Silva Herzog, Jesús, El agrarismo mexicano y la Reforma Agraria, Fondo de Cultura Económica, México, 1964.

Tor regrosa, María Luisa, El ejido colectivo San Ignacio Rio Muerto en el marco de la Reforma Agraria Integral (Tesis de licenciatura), Facultad de Ciencias Políticas. UNAM, México, 1980.

TORRES, MARio, "Una aplicación empírica de un enfoque de reproducción social" (ed. mimeografiada). PISPAL-EI Colegio de México, Mé$\mathrm{xico}, 1981$.

TEPICHT JERZY. Marxisme et agriculture, le paysan polonais, Armand Colin, París, 1973.

WOLF, ERIK, Las luchas campesinas del siglo XX, Siglo XXI Editores, México, 1972.

YouNG, KATE, "Formas de apropiación y la división sexual del trabajo" en Magdalena León (comp.), Las Trabajadoras del agro (vol. II), ACEP, Bogotá, 1982.

Yunes, Antonio, "Una evaluación de los estudios sobre eficiencia de las explotaciones agrícolas con base en la agricultura mexicana", Demografia y Economia, vol. XI, núm. 1, El Colegio de México, México, 1977. 